\title{
EMT in Liver Fibrosis
}

\author{
V. Sterzer • M. Alsamman · T. Bretag • \\ D. Scholten
}

Published online: 17 September 2014

(C) Springer Science+Business Media New York 2014

\section{Introduction}

Liver Fibrosis and Origin of Myofibroblasts

Liver cirrhosis is the outcome of multiple causes of chronic liver injury such as viral inflammation (HBC, HCV), alcoholic or metabolic injury (ASH, NASH), or occlusive diseases of the small bile ducts (PSC, PBC). Histologically cirrhotic/fibrotic livers are characterized by excessive accumulation of collagen type I and other extracellular matrix (ECM) proteins [1]. Myofibroblasts are characterized immunophenotypically by a spindle or stellate shape, pale eosinophilic cytoplasm, expression of abundant pericellular matrix, and fibrotic genes (vimentin, $\alpha$-smooth muscle actin ( $\alpha$-SMA), nonmuscle myosin, fibronectin) [2]. The cellular ultrastructure is defined by prominent rough endoplasmic reticulum (rER), a Golgi apparatus producing collagen, peripheral myofilaments, fibronexus (no lamina), and gap junctions [2]. Myofibroblasts are implicated in wound healing and fibroproliferative disorders [3-5]. Studies of fibrogenesis conducted in different organs strongly suggest that resident myofibroblasts are the primary source of ECM [6]. In response to fibrogenic stimuli, such as TGF- $\beta 1$, myofibroblasts in all tissues express $\alpha$-SMA, and secrete ECM (fibronectin, collagen type I and III), obtain high contractility and change their phenotype (production of the stress fibers) [7]. Classical myofibroblasts differentiate from a mesenchymal lineage and, therefore, lack expression of lymphoid markers such as CD45 or CD34. Sustained injury may trigger (trans) differentiation of myofibroblasts from other cellular sources, including hepatic stellate cells (HSCs) [1]. Upon liver injury, HSCs transdifferentiate from a quiescent vitamin A storing phenotype into activated myofibroblasts, losing vitamin A droplets, and producing multiple profibrotic
V. Sterzer, M. Alsamman and T. Bretag have equally contributed to this work.

V. Sterzer · M. Alsamman - T. Bretag $\cdot$ D. Scholten $(\bowtie)$

Department of Medicine III, RWTH Aachen University

Hospital, 52074 Aachen, Germany

e-mail: dscholten@ukaachen.de 
cytokines such as TGF- $\beta$ [8]. However, quiescent HSCs are not the only source of myofibroblasts in the injured liver. Despite of extensive studies, the origin of fibrogenic myofibroblasts is still unresolved and remains a subject for scientific debates.

Although it is believed that upon differentiation, all myofibroblasts acquire common characteristics, they originate from different sources dependent on etiology of fibrosis [9*•]. Next to activated HSC contributing to the pool of myofibroblasts portal fibroblasts [10], interphase (septal) myofibroblasts and bone marrow-derived fibrocytes $[11,12 \bullet \cdot$ give rise to myofibroblasts in the injured liver. Identification of the myofibroblast composition arising in fibrotic tissue in correlation with the cause of underlying chronic liver injury will provide new insights in pathogenesis of fibrogenic liver disease. Since myofibroblasts are the major source of collagen type I and ECM deposition in all fibrosing organs, they are the primary target for antifibrotic therapy.

\section{The concept of EMT}

Previous studies in kidney [13], lung [14], heart [15], and liver have implicated parenchymal cells to contribute to fibrogenesis by giving rise to myofibroblasts. Thus, they suggested that resident epithelial cells may undergo a process called epithelial-to-mesenchymal transition (EMT) in response to injury. During this process, mature epithelial cells change their phenotype into functional mesenchymal cells. They lose characteristics such as cell polarity, epithelial marker (e.g. cytokeratin-19, cytokeratin-7, E-cadherin) and tight junction protein (e.g., zonula occludens-1) expression, detach from the epithelial layer, increase their motility, and obtain a myofibroblastic phenotype [16]. During many stages of development, EMT is a common process and plays an important role in embryogenesis [17]. However, in adult tissues EMT usually is tightly controlled because such cell plasticity can lead to cancer development and is required for cancer metastasis [18, 19]. Depending on the biological and pathophysiological background, EMT can be observed in three different conditions. It is important and well studied during development and embryogenesis (Type 1). It can be seen in tissue remodeling due to wound healing and repair (Type 2) and it describes the invasion of cancer cells and plays a crucial role in development of metastasis (Type 3) [20-23].

EMT in chronic injury was first characterized in kidney fibrosis due to unilateral ureter obstruction. Using genetic cell fate-tracking techniques (gGT-LacZ transgenic mice), tubular epithelial cells could be labeled and identified in fibrotic kidneys. In this study, Iwano et al. [24] demonstrated that renal interstitial fibroblasts originate in more than $30 \%$ from EMT. Additionally several studies described EMT in fibrosis in chronic lung injury [14], rheumatoid arthritis [25], and retinopathy [26]. In adult, liver EMT has been linked to chronic inflammation, cancer, primary biliary fibrosis, and nonalcoholic fatty liver disease in patients and rodents [27, 28]. However, these studies, especially in human patients, relied mostly on immunohistochemical stainings. Co-localization of epithelial and myofibroblastic markers in the same cell usually lead to the conclusion that these cells had shifted from an epithelial origin into a myofibroblastic phenotype. Hence, immunohistochemistry analysis has several limitations, it does not allow monitoring the dynamic changes in the differentiating cells in the injured tissues, but provides a "snapshot" of proteins expressed at the time of the study. Therefore, many cellular features characteristic for naive or quiescent phenotype as well as previously expressed proteins cannot be detected by this method, indicating that the cellular origin of myofibroblasts might not be always established using immunohistochemistry techniques $[17,29]$. In support of this notion, many observations based on coexpression of specific epithelial and mesenchymal markers at one given timepoint in injured, inflamed, or scarred tissues actively undergoing wound healing and remodeling must be carefully evaluated and confirmed using complimentary or alternative approaches. Thus, in the recent years, the Cre-LoxP-based genetic linage tracing became a method of choice to study the cell fate mapping of fibrogenic myofibroblasts, and in combination with immunohistochemical and functional analyses can be used to dissect the original cellular phenotype and injury-induced changes. The proposed complimentary approach is particularly critical to study the cells potentially undergoing EMT. Due to the difficulties and limitations of existing techniques outlined above, several contradicting studies have been published and instigated an ongoing debate on the relevance of EMT in fibrosis. Although the role of EMT in fibrogenesis is still unresolved, several recently published studies utilizing in vivo genetic linage-tracing techniques have disproved the role of EMT in liver and kidney fibrosis and seriously questioned the contribution of EMT to fibrogenesis of other parenchymal organs [30]. First evidence of EMT in fibrotic injury was provided by studies in fibrotic kidneys and lung fibrosis [24, 31]. These concepts and results then have been adopted to liver fibrosis. In vitro data supported these concepts. Cell culture studies of primary cholangiocytes and hepatocytes [32] clearly showed evidence that these cells undergo a change in phenotype and gene expression exhibiting mesenchymal cell features. This was even more pronounced after incubation with TGF- $\beta$, a cytokine closely associated with liver fibrosis, tissue remodeling, and EMT [29]. In vivo studies used immunohistochemical techniques both in murine models of liver fibrosis and patients with chronic liver 
disease and showed the co-expression of mesenchymal markers (fibroblast-specific protein 1 [FSP1], $\boldsymbol{\alpha}$-SMA, vimentin, desmin) with typical epithelial markers (keratin19 for cholangiocytes and albumin for hepatocytes). Finally at least one study used genetic cell fate tracking in fibrotic mouse liver to show that cells originally expressing albumin (hepatocytes) exhibited an FSP- $1^{+}$myofibroblast like phenotype after liver injury [33]. However, the functional relevance of EMT (no detection of $\alpha$-SMA- or collagen expression) remained unclear and identification of the myofibroblast origin often failed. To clearly investigate EMT in liver fibrosis robust irreversible lineage-tracing studies have been performed, well defined, and established markers for epithelial cells, myofibroblasts, and ongoing EMT have been analyzed and cell function (i.e., ECM production) have been taken into account. Recently, three studies applied these concepts to their experimental setups, finally questioning the role of EMT in liver fibrosis. Taura et al. used the well established and highly efficient albu$\min ^{\text {Cre }}$ mouse to mark all hepatocytes [34]. In our own work, we labeled cholangiocytes using tamoxifen-inducible cytokeratin-19-Cre-ERT mice (K19 ${ }^{\text {CreERT }}$ ) mice [35]. Finally $\mathrm{Chu}$ et al. crossed the alpha-fetoprotein-Cre mice $\left(\mathrm{AFP}^{\mathrm{Cre}}\right.$ mice) with the ROSA26 ${ }^{\mathrm{YFP}}$ reporter mouse labeling any cell ever expressing AFP (cholangiocytes, hepatocytes) [36••]. Even if there is in vitro evidence that hepatocytes and cholangiocytes can exhibit markers of mesenchymal cells after TGF- $\beta$ stimulation these studies come to different results in vivo. All three studies using three independent strains of Cre-expressing mice in hepatic epithelial cells as well as different experimental methods (fluorescence-activated cell sorting, immunofluorescence to detect myofibroblast markers, and $\beta$-galactosidase enzymatic activity) demonstrated that hepatic epithelial cells do not undergo EMT in the damaged liver and do not give rise to the pool of activated myofibroblasts in experimental liver fibrosis. Furthermore, Östereicher et al. [37••] showed that FSP-1, a common marker widely used to identify cells undergoing EMT, is expressed by nonfibroblast cells in the liver, including a subset of monocytes, and does not colocalize with myofibroblasts. The current review will summarize the recent data on the role of EMT in fibrogenesis if parenchymal organs.

\section{Fibroblast-Specific Protein-1 (FSP-1): A Marker of EMT}

FSP-1 was described in 1995 by Strutz et al. [38] as a murine fibroblast-specific protein that belongs to the calmodulinS100-troponin C superfamily of intracellular calcium-binding proteins. Members of this protein family take part in microtubulus formation [39], interactions between cytoskelet and cell membrane [40, 41], calcium signaling [42] as well as cell growth, cell cycle, and regeneration [43]. FSP-1 interacts with cell structure filaments like nonmuscle myosin II, tropomyosin, tubulin, and actin [44-46]. Furthermore, FSP-1 can induce a migratory or metastatic phenotype when transfected into nonmetastatic cells in vitro [47] suggesting that FSP-1 is associated with mesenchymal cell shape and motility. Transfecting FSP-1 into tubular epithelium leads to the expression of typical features of EMT such as reduction of cell adhesion and denovo expression of vimentin [38]. FSP-1 is expressed in tissue undergoing remodeling in response to injury [48], and it was shown that cells of epithelial origin, mesangial cells, or embryonic endoderm lack FSP-1 expression. Because of this distinctive cellular distribution and its functional properties in mesenchymal cells FSP-1 expression is usually seen as a marker for cells undergoing EMT [49].

\section{Kidney Fibrosis}

Humphreys et al. [50] reassessed the concept of EMT in kidney fibrosis. In this study, the authors used tamoxifeninducible cell specific Cre mice and crossed them to two different reporter mice. Utilizing two models of kidney fibrosis, the authors were able to trace epithelial cells in the fibrotic nephrons. In this study, the authors demonstrated that interstitial myofibroblasts do not originate from nephron epithelial cells. The authors suggest that similar to hepatic fibrosis (in which liver resident HSCs provide a major source of hepatic myofibroblasts in response to injury [51]), almost all myofibroblasts in fibrotic kidney derive from pericytes (FoxD1-expressing cells).

\section{Lung Fibrosis}

Many studies show that EMT is involved in the pathogenesis of various lung diseases ranging from developmental disorders, lung fibrosis to pulmonary cancer. However, the importance and contribution of EMT in the pathogenesis fibrotic injury in chronic lung conditions such as asthma, COPD, and inflammatory lung injury are controversially discussed. Many studies find cells expressing typical features for EMT regardless of the underlying injury [52-54]. However, most studies rely on the co-expression of epithelial and mesenchymal markers in response to lung injury, either analyzed by immunofluorescence or serial sections. However, only few studies use genetic cell fate tracking to analyze cells undergoing EMT. Tanjore et al. [55] used mice expressing Cre recombinase under the control of the surfactant protein $\mathrm{C}$ promoter to genetically label cells of epithelial origin. These mice were subjected to bleomycin-induced fibrotic injury. Tissue sections were analyzed for cells coexpressing $\mathrm{S} 100 \mathrm{~A}^{+}$ 
and $\beta$-Gal finding that approximately one-third of the $\mathrm{S} 100 \mathrm{~A}^{+}{ }^{+}$fibroblasts were derived from lung epithelium two weeks after bleomycin administration. Next, outgrowth fibroblast culture studies were performed from R26RosaStop-LacZ-SPC-Cre reporter mouse lungs after two weeks bleomycin-induced injury. However, outgrowth fibroblasts at from R26Rosa-Stop-LacZ-SPC-Cre demonstrated rare X-Gal-positive cells, whereas positive controls (R26RosaStop-LacZ-S100A4-Cre lung fibroblasts) were uniformly $\mathrm{X}$-Gal positive. The authors conclude that EMT-derived lung fibroblasts may exhibit a different phenotype from fibroblasts of other origin. EMT-derived lung fibroblasts seem to have a decreased ability to proliferate in outgrowth culture. Therefore, EMT results in a substantial subset of S100A4 ${ }^{+}$lung fibroblasts in bleomycin-induced lung fibrosis. Thus, the impact of these cells on the progression of pulmonary fibrosis needs to be further investigated.

\section{Liver Fibrosis}

Original studies by Zeisberg et al. [33] have suggested that EMT generates significant numbers of myofibroblasts in fibrotic liver. This study was based on genetic labeling of albumin $^{+}$hepatocytes (using Albumin-Cre mice crossed LacZ-reporter mice) that a population of hepatic FSP- $1^{+}$ fibroblasts is originated from mature hepatocytes. However, Albumin ${ }^{+}$FSP- $1^{+}$fibroblasts expressed nearly no $\alpha$ SMA $(<10 \%)$, thereby questioning the functional role of these cells. A study performed by Taura et al. [34] further clarified and rather contradicted these data. The cell fate mapping of hepatocytes was monitored in response to toxic liver injury (carbontetrachloride, $\mathrm{CCl}_{4}$ )-injured triple transgenic mice generated by crossing of albumin-Cre mice to ROSA26-LacZ-reporter mice and collagen- $\alpha 1$ (I)-GFP mice. While expression of collagen- $\alpha 1$ (I)-GFP transgene allowed detecting emerging myofibroblasts in fibrotic liver, genetic labeling of heptocytes was designed to study possible transition of damaged hepatocytes. Hence, the authors could not detect transition of $\mathrm{LacZ}^{+}$hepatocytes into collagen-expressing myofibroblasts in vivo in $\mathrm{CCl}_{4}$ induced mice, suggesting that EMT in hepatocytes does not contribute to collagen producing myofibroblasts in fibrotic liver. Interestingly, Taura et al. [34] supported the previous observations that EMT can occur in TGF- $\beta 1$-stimulated hepatocytes and may result from in vitro artifacts. Similar to that, Humphreys et al. [50] detected markers of EMT in isolated proximal tubular cells and hepatocytes upon in vitro stimulation with TGF- $\beta 1$.

Another cellular population, which has been suggested to undergo EMT in response to liver injury, are cholangiocytes. Cholangiocytes are a heterogenuous epithelial cell population with many highly specialized cell functions [56]. In injured liver, especially in cholestatic liver disease, cholangiocytes proliferate, activate, and change their morphology and gene expression profile thereby modulating the development of fibrosis and inflammation. Next to myofibroblasts, reactive cholangiocytes express important profibrogenic cytokines such as platelet-derived growth factor-BB (PDGF-BB), ligands of the Hedgehog pathway, connective tissue growth factor (CTGF), and/or transforming growth factor beta (TGF- $\beta 2$ ) [57]. These cytokines can activate myofibroblasts and further induce cholangiocyte proliferation. The compartment of reactive cholangiocytes also includes bipotent adult hepatic progenitor cells (oval cells) capable of differentiating into bile duct epithelal cells and/or hepatocytes under specific circumstances [58]. Due to this plasticity, precursors of cholangiocytes have been suggested to undergo EMT in response to liver injury, specifically cholestatic liver fibrosis. Our group investigated if cholangiocyte undergo EMT in response to bile duct ligation (BDL). To address this question, cholangiocyte-specific cytokeratin 19-CreERT mice (K19 ${ }^{\text {CreERT }}$ mice) [59], in which tamoxifen-inducible Cre-ERT was knocked into the endogenous K19 locus, were crossed to ROSA26 ${ }^{\mathrm{YFP}}$ reporter mice [35]. Tamoxifen-inducible K19-Cre knockin mice provide important advantages for cell fate-tracking experiments. Although specific homologous recombination to generate the knockin mice is generally low, overexpression of Cre within the specific genetic locus is usually controlled by promoterspecific regulatory elements. Furthermore, generation of inducible Cre-expressing mice does allow to target cell specific gene labeling in adult mice, ruling out effects of promotor activation during development [30]. The duration of gene expression and cell labeling can be also controlled by repetitive tamoxifen administration.

Thus, cholangiocytes were irreversibly labeled by YPF expression in these mice prior to BDL-induced liver injury. Hence, their cell fate was monitored in response to cholestatic liver fibrosis, and injured cholangiocytes were analyzed for expression of myofibroblast markers such as $\alpha$-SMA. However, none of $\mathrm{YFP}^{+}$cholangiocytes up-regulated $\alpha$-SMA or any other markers of myofibroblasts in livers of BDL-injured mice, suggesting that it is unlikely that cholangiocytes undergo EMT in response to cholestatic liver injury which is closely associated to cholangiocyte activation.

Furthermore, mice expressing Cre under control of human glial fibrillary acidic protein (GFAP) promotor have been reported to specifically label HSCs but not cholangiocytes [60], while mice expressing Cre under control of the fibroblast-specific protein (FSP-1) promoter were shown to be specific for cells undergoing EMT. Following induction of liver fibrosis tissues was analyzed for co-expression of YFP and myofibroblast markers ( $\alpha$-SMA, desmin). Even though $\mathrm{YFP}^{+}$staining was often observed in 
close proximity to $\alpha-\mathrm{SMA}^{+}$or desmin ${ }^{+}$staining, a co-localization of all these markers in the same cells has never been detected.

The opposite to the EMT phenomenon known as mesenchymal-to-epithelial transition (MET) has been also described and was also implicated in pathogenesis of liver fibrosis. MET has been suggested to play a role in tissue repair and remodeling. Cell fate mapping of $\mathrm{YFP}^{+} \mathrm{HSCs}$ in livers of BDL-injured GFAP-Cre (crossed to Rosa26 ${ }^{\text {YFP }}$ reporter) mice had demonstrated that $\mathrm{YFP}^{+}$cells did not give rise to pan-cytokeratin ${ }^{+}$hepatocytes and $\mathrm{K}_{1}{ }^{+}$(and $\mathrm{TROMA}^{+}$) cholangiocytes [35]. Furthermore, $\mathrm{YFP}^{+} \mathrm{HSCs}$ have never up-regulated epithelial marker like E-cadherin in the course of liver fibrosis. Taken together, this study did not find either evidence of EMT nor evidence of MET in livers of mice subjected to liver fibrosis [35].

The most critical evidence for the lack of EMT in fibrotic liver was provided by study by $\mathrm{Chu}$ et al. [36••]. The authors used alpha-fetoprotein AFP $^{\text {Cre mice (crossed }}$ with Rosa $26^{\text {YFP }}$ reporter mice, in which all liver epithelial cells and their precursors (hepatocytes, cholangiocytes, and their bipotential progenitors) were irreversibly labeled by YFP expression. Similar to the findings reported by Taura et al. and Humphreys et al., primary YFP expressing cholangiocytes were able to undergo EMT in vitro after treatment with TGF- $\beta 1$ or treatment with TGF- $\beta 1$ plus tumor necrosis factor- $\alpha$. Cultured cholangiocytes changed their morphology and exhibited fibroblast-like shape, showed intracellular expression of E-cadherin and up-regulated $\alpha$-smooth muscle actin ( $\alpha$-SMA). However, none of the $\mathrm{YFP}^{+}$hepatocytes, cholangiocytes, or their precursors expressed markers of myofibroblasts, including $\alpha$-SMA, vimentin, or procollagen $1 \alpha 2$. Thus, using genetic cell fate mapping in mice subjected to three well-established models of liver injury (BDL (2, 4, and 8 weeks), $\mathrm{CCl}_{4}$ treatment for 3 weeks and 3,5-diethoxycarbonyl-1,4-dihydrocollidine (DDC; 2 and 3 weeks), epithelial cells did not upregulate mesenchymal markers FSP-1 (S100A4), vimentin, $\alpha$-SMA, or pro-collagen $1 \alpha 2$ in vivo.

\section{The Role of FSPI as a Marker of EMT}

Another recent study by Östereicher et al. [37••] investigated the role of fibroblast-specific protein-1 (FSP-1) expression in EMT. In several studies, FSP-1 $\left(\mathrm{Ca}^{+}{ }_{-}\right.$ binding S100 protein) was identified in fibroblasts but not in cells of epithelial origin, mesangial cells or embryonic endoderm [38]. Because it is expressed in organs undergoing tissue-remodeling FSP-1 was often seen as proof of EMT [61]. However, the functional role of FSP-1 positive cells in fibrotic livers remained unclear. As expected the work of Östereicher et al. showed increased numbers of FSP-1 positive cells in fibrotic livers of humans and rodents. These cells were located in fibrotic septa and showed the same spacial location as activated myofibroblasts. Surprisingly the authors show that activated hepatic myofibroblasts do not express FSP-1. Using a $\mathrm{Col}^{\mathrm{GFP}}$ mouse in which the collagen $\alpha 1(\mathrm{I})$ promoter/enhancer drives green fluorescent protein (GFP) expression the authors show that GFP expressing myofibroblasts do not colocalize with FSP-1 positive cells in injured liver. Vice versa FSP-1 ${ }^{\text {GFP }}$ mice, in which the FSP-1 promoter drives GFP expression, were subjected to liver injury. Again, GFP-positive cells show no co-staining with $\alpha$-SMA or desmin, typical markers of activated myofibroblasts. To rule out that FSP-1 expressing cells are precursors for myofibroblasts in fibrotic liver and FSP-1 expression disappears before the upregulation of typical myofibroblast markers the authors used genetic cell labeling by Cre-LoxP recombination. By crossing FSP-1 ${ }^{\text {Cre }}$ mice to ROSA26 ${ }^{\text {YFP }}$ reporter mice cells that have expressed FSP-1 during development or differentiation but lack FSP-1 expression at the time of analysis could be identified. Again no colocalization of GFP-positive cells with $\alpha$-SMA or desmin expression could be shown. Gene expression profiling finally placed FSP-1-positive cells in fibrotic liver close to peritoneal macrophages stimulated with zymosan. The authors show that FSP-1 expressing cells isolated from fibrotic livers expressed genes typical for macrophages and cells of dendritic lineage (CD68, Nramp1, Soat1, CD63, CD83, CD93, Clec4d, Clec4b1, Clec4n, Clec7a, and p22phox). Moreover, genes involved in innate immunity (CD14, TLR4, TLR2, TLR7, TLR8) were up-regulated in these cells suggesting that FSP- $1^{+}$cells detected in the injured liver may belong to myeloid-monocytic lineage [62]. These data question the role of FSP-1 as a marker for EMT in fibrotic liver and emphasize the importance of well-defined cellular markers as well as characterization of cell function in EMT.

\section{Conclusion}

Recent studies have questioned the contribution of EMT to liver fibrosis. However, the role of EMT in liver fibrosis cannot be completely ruled out. Thus, more severe injury to epithelial liver cells (compared to BDL) such as observed in response to alcoholic liver disease, might promote EMT in these situations more readily. Furthermore, in contrast to mouse models of liver disease, where animals are subjected to liver injury for 3 weeks -3 month chronic liver injury in human patients is a long-lasting disease progressing over several years. This might influence pathophysiology and lead to mechanisms like EMT which are not seen in rodent models of liver fibrosis. After all many studies demonstrate that injured epithelial cells show a certain amount of 
plasticity, producing hedgehog ligands (hepatocytes) [63] or TGF- $\beta$ (cholangiocytes) [64] thereby activating HSCs.

Even though there is no current evidence that myofibroblasts originate from hepatic epithelial cells, epithelial liver cells show a high amount of plasticity in response to liver injury. As in other models of tissue remodeling the concept of EMT in response to liver injury and regeneration remains an interesting approach to understand cell differentiation and transition.

\section{Compliance with Ethics Guidelines}

Conflict of Interest V. Sterzer, M. Alsamman, T. Bretag, D. Scholten declare no conflicts of interest.

Human and Animal Rights and Informed Consent This article does not contain any studies with human or animal subjects performed by any of the authors.

\section{References}

Papers of particular interest, published recently, have been highlighted as:

-• Of major importance

1. Bataller R, Brenner DA (2005) Liver fibrosis. J Clin Invest 115(2):209-218

2. Eyden B (2008) The myofibroblast: phenotypic characterization as a prerequisite to understanding its functions in translational medicine. J Cell Mol Med 12(1):22-37

3. Majno $G$ et al (1971) Contraction of granulation tissue in vitro: similarity to smooth muscle. Science 173(996):548-550

4. Gabbiani G, Ryan GB, Majne G (1971) Presence of modified fibroblasts in granulation tissue and their possible role in wound contraction. Experientia 27(5):549-550

5. Schurch W, Seemayer TA, Gabbiani G (1998) The myofibroblast: a quarter century after its discovery. Am J Surg Pathol 22(2):141-147

6. Kisseleva T, Brenner DA (2008) Mechanisms of fibrogenesis. Exp Biol Med (Maywood) 233(2):109-122

7. Parola M, Marra F, Pinzani M (2008) Myofibroblast-like cells and liver fibrogenesis: emerging concepts in a rapidly moving scenario. Mol Aspects Med 29(1-2):58-66

8. Kisseleva T, Brenner DA (2007) Role of hepatic stellate cells in fibrogenesis and the reversal of fibrosis. J Gastroenterol Hepatol 22(Suppl 1):S73-S78

9. • Iwaisako K et al. (2014) Origin of myofibroblasts in the fibrotic liver in mice. Proc Natl Acad Sci USA 111.32:E3297-E3305. In two models of liver fibrosis the origin of distinct populations of myofibroblasts is investigated and their contribution to hepatic fibrosis is quantified. Whereas hepatic stellate cells are the major source for fibrogenic myofibroblasts in $\mathrm{CCl}_{4}$ induced liver injury activated portal fibroblasts give rise to more than $70 \%$ of myofibroblasts in cholestatic liver injury at the onset of injury

10. Dranoff JA, Wells RG (2010) Portal fibroblasts: underappreciated mediators of biliary fibrosis. Hepatology 51(4):1438-1444

11. Kisseleva $T$ et al (2006) Bone marrow-derived fibrocytes participate in pathogenesis of liver fibrosis. J Hepatol 45(3):429-438
12. $\bullet$ Scholten D et al. (2011) Migration of fibrocytes in fibrogenic liver injury. Am J Pathol 179(1):189-98. Bone marrow derived fibrocytes contribute to the pool of hepatic myofibroblasts in response to liver injury. In response to liver injury but also during liver regeneration fibrocytes migrate from bone marrow to the injured liver

13. Zeisberg EM et al (2008) Fibroblasts in kidney fibrosis emerge via endothelial-to-mesenchymal transition. J Am Soc Nephrol 19(12):2282-2287

14. Willis BC, Borok Z (2007) TGF-beta-induced EMT: mechanisms and implications for fibrotic lung disease. Am J Physiol Lung Cell Mol Physiol 293(3):L525-L534

15. Zeisberg EM et al (2007) Endothelial-to-mesenchymal transition contributes to cardiac fibrosis. Nat Med 13(8):952-961

16. Choi SS, Diehl AM (2009) Epithelial-to-mesenchymal transitions in the liver. Hepatology 50(6):2007-2013

17. Zeisberg M, Neilson EG (2009) Biomarkers for epithelial-mesenchymal transitions. J Clin Invest 119(6):1429-1437

18. Moustakas A, Heldin CH (2007) Signaling networks guiding epithelial-mesenchymal transitions during embryogenesis and cancer progression. Cancer Sci 98(10):1512-1520

19. Huber MA, Kraut N, Beug H (2005) Molecular requirements for epithelial-mesenchymal transition during tumor progression. Curr Opin Cell Biol 17(5):548-558

20. Kalluri R, Weinberg RA (2009) The basics of epithelial-mesenchymal transition. J Clin Invest 119(6):1420-1428

21. Kalluri R (2009) EMT: when epithelial cells decide to become mesenchymal-like cells. J Clin Invest 119(6):1417-1419

22. Acloque $\mathrm{H}$ et al (2009) Epithelial-mesenchymal transitions: the importance of changing cell state in development and disease. J Clin Invest 119(6):1438-1449

23. Jou J, Diehl AM (2010) Epithelial-mesenchymal transitions and hepatocarcinogenesis. J Clin Invest 120(4):1031-1034

24. Iwano $\mathrm{M}$ et al (2002) Evidence that fibroblasts derive from epithelium during tissue fibrosis. J Clin Invest 110(3):341-350

25. Steenvoorden MM et al (2006) Transition of healthy to diseased synovial tissue in rheumatoid arthritis is associated with gain of mesenchymal/fibrotic characteristics. Arthritis Res Ther $8(6):$ R165

26. Lee $\mathrm{H}$ et al (2007) The role of gremlin, a BMP antagonist, and epithelial-to-mesenchymal transition in proliferative vitreoretinopathy. Invest Ophthalmol Vis Sci 48(9):4291-4299

27. Ikegami T, Zhang Y, Matsuzaki Y (2007) Liver fibrosis: possible involvement of EMT. Cells Tissues Organs 185(1-3):213-221

28. Nitta $T$ et al (2008) Murine cirrhosis induces hepatocyte epithelial mesenchymal transition and alterations in survival signaling pathways. Hepatology 48(3):909-919

29. Zavadil J, Bottinger EP (2005) TGF-beta and epithelial-tomesenchymal transitions. Oncogene 24(37):5764-5774

30. Popov Y, Schuppan D (2010) Epithelial-to-mesenchymal transition in liver fibrosis: dead or alive? Gastroenterology 139(3):722-725

31. Chilosi $\mathrm{M}$ et al (2003) Aberrant Wnt/beta-catenin pathway activation in idiopathic pulmonary fibrosis. Am J Pathol 162(5):1495-1502

32. Kaimori A et al (2007) Transforming growth factor-beta1 induces an epithelial-to-mesenchymal transition state in mouse hepatocytes in vitro. J Biol Chem 282(30):22089-22101

33. Zeisberg $M$ et al (2007) Fibroblasts derive from hepatocytes in liver fibrosis via epithelial to mesenchymal transition. J Biol Chem 282(32):23337-23347

34. Taura K et al (2010) Hepatocytes do not undergo epithelialmesenchymal transition in liver fibrosis in mice. Hepatology 51(3):1027-1036

35. Scholten D et al (2010) Genetic labeling does not detect epithelial-to-mesenchymal transition (EMT) of cholangiocytes in liver fibrosis in mice. Gastroenterology 139(3):987-998 
36. • Chu A.S et al. (2011) Lineage tracing demonstrates no evidence of cholangiocyte epithelial-to-mesenchymal transition in murine models of hepatic fibrosis. Hepatology 53(5):1685-95. Genetic cell fate tracking of $\mathrm{AFP}^{+}$cells (cholangiocytes, hepatocytes) reveals no contribution of EMT of hepatocytes and cholangiocytes in murine models of hepatic fibrosis

37. • Osterreicher C.H et al. (2011) Fibroblast-specific protein 1 identifies an inflammatory subpopulation of macrophages in the liver. Proc Natl Acad Sci USA 108(1):308-13. Fibroblast-specific protein 1 (FSP-1) is upregulated on epithelial cells undergoing EMT. However in injured liver myofibroblasts do not express FSP-1 but FSP-1 is identifies an inflammatory subpopulation of macrophages. This questions the pivotal role of FSP-1 as a general marker of EMT

38. Strutz $F$ et al (1995) Identification and characterization of a fibroblast marker: FSP1. J Cell Biol 130(2):393-405

39. Donato R, Battaglia F, Cocchia D (1987) Characteristics of the effect of S-100 proteins on the assembly-disassembly of brain microtubule proteins at alkaline $\mathrm{pH}$ in vitro. Cell Calcium 8(4):299-313

40. Fano $G$ et al (1995) The S-100: a protein family in search of a function. Prog Neurobiol 46(1):71-82

41. Gerke V, Weber K (1985) The regulatory chain in the p36-kd substrate complex of viral tyrosine-specific protein kinases is related in sequence to the S-100 protein of glial cells. EMBO J 4(11):2917-2920

42. Santamaria-Kisiel L, Rintala-Dempsey AC, Shaw GS (2006) Calcium-dependent and -independent interactions of the S100 protein family. Biochem J 396(2):201-214

43. Heizmann CW (2002) The multifunctional S100 protein family. Methods Mol Biol 172:69-80

44. Ford HL, Zain SB (1995) Interaction of metastasis associated Mts1 protein with nonmuscle myosin. Oncogene 10(8):1597-1605

45. Takenaga $\mathrm{K}$ et al (1994) Binding of pEL98 protein, an S100related calcium-binding protein, to nonmuscle tropomyosin. J Cell Biol 124(5):757-768

46. Wang $G$ et al (2000) Interaction in vivo and in vitro of the metastasis-inducing S100 protein, S100A4 (p9Ka) with S100A1. J Biol Chem 275(15):11141-11146

47. Davies BR et al (1993) Induction of the metastatic phenotype by transfection of a benign rat mammary epithelial cell line with the gene for $\mathrm{p} 9 \mathrm{Ka}$, a rat calcium-binding protein, but not with the oncogene EJ-ras-1. Oncogene 8(4):999-1008

48. Schneider $M$ et al (2007) S100A4 is upregulated in injured myocardium and promotes growth and survival of cardiac myocytes. Cardiovasc Res 75(1):40-50
49. Okada H et al (1997) Early role of Fsp1 in epithelial-mesenchymal transformation. Am J Physiol 273(4 Pt 2):F563-F574

50. Humphreys BD et al (2010) Fate tracing reveals the pericyte and not epithelial origin of myofibroblasts in kidney fibrosis. Am J Pathol 176(1):85-97

51. Friedman SL (2008) Hepatic stellate cells: protean, multifunctional, and enigmatic cells of the liver. Physiol Rev 88(1): $125-172$

52. Chen T et al (2014) Epithelial-mesenchymal transition involved in pulmonary fibrosis induced by multi-walled carbon nanotubes via TGF-beta/smad signaling pathway. Toxicol Lett 226(2): $150-162$

53. Ohbayashi $M$ et al (2014) Involvement of epithelial-mesenchymal transition in methotrexate-induced pulmonary fibrosis. J Toxicol Sci 39(2):319-330

54. Shen HJ et al (2014) Cigarette smoke-induced alveolar epithelialmesenchymal transition is mediated by Rac1 activation. Biochim Biophys Acta 1840(6):1838-1849

55. Tanjore $\mathrm{H}$ et al (2009) Contribution of epithelial-derived fibroblasts to bleomycin-induced lung fibrosis. Am J Respir Crit Care Med 180(7):657-665

56. Alpini G et al (1998) Heterogeneity of the proliferative capacity of rat cholangiocytes after bile duct ligation. Am J Physiol 274(4 Pt 1):G767-G775

57. Lazaridis KN, Strazzabosco M, Larusso NF (2004) The cholangiopathies: disorders of biliary epithelia. Gastroenterology 127(5):1565-1577

58. Walkup MH, Gerber DA (2006) Hepatic stem cells: in search of. Stem Cells 24(8):1833-1840

59. Means AL et al (2008) A CK19(CreERT) knockin mouse line allows for conditional DNA recombination in epithelial cells in multiple endodermal organs. Genesis 46(6):318-323

60. Yang L et al (2008) Fate-mapping evidence that hepatic stellate cells are epithelial progenitors in adult mouse livers. Stem Cells 26(8):2104-2113

61. Okada H et al (1997) Early role of Fsp1 in epithelial-mesenchymal transformation. Am J Physiol 273(4):F563-F574

62. Schuster C, Lefevre M, Baumert TF (2011) Triglyceride synthesis and hepatitis $\mathrm{C}$ virus production: identification of a novel host factor as antiviral target. Hepatology 53(3):1046-1048

63. Jung $Y$ et al (2010) Signals from dying hepatocytes trigger growth of liver progenitors. Gut 59(5):655-665

64. Patsenker E et al (2008) Inhibition of integrin alphavbeta6 on cholangiocytes blocks transforming growth factor-beta activation and retards biliary fibrosis progression. Gastroenterology 135(2):660-670 